Rev. Biol. Trop., 48(2/3): 539-554, 2000

www.ucr.ac.cr www.ots.ac.cr www.ots.duke.edu

\title{
Basidiomicetos resupinados de Costa Rica. Especies nuevas o raras de Atractiellales (Auriculariales s.l.), Exidiaceae, Sirobasidiaceae y Tremellaceae
}

\author{
Liuba Kisimova-Horovitz ${ }^{1}$, Franz Oberwinkler ${ }^{1}$ y Luis D. Gómez P. ${ }^{2}$ \\ 1 Botanisches Institut, Mykologie, Universität Tübingen, Alemania, \\ 2 Academia Nacional de Ciencias, y Estación Biológica Las Cruces, O.T.S., Coto Brus, Costa Rica , fax (506)7733665; \\ ldgomez@hortus.ots.ac.cr).
}

Recibido 10-VIII-1999. Corregido 27-III-2000. Aceptado 30-III-2000.

\begin{abstract}
Species of Atractiellales (Auriculariaceae s.l.), Exidaceae, Sirobasidaceae and Tremellaceae are reported as new for Costa Rica or as new to science, Tremella coalescens L.S. Olive, Sirobasidium minutum Kisim., Oberw. \& Gómez sp. nov., Heterochaete vitrea Kisim., Oberw. \& Gómez sp. nov., Exidiopsis mucedinea (Pat.) K.Wells, Helicogloea aurea Baker, Saccoblastia sphaerospora Möller and Occultifur internus (L.S. Olive) Oberw. All the new species are described and illustrated. Since the original material collected in Brazil by Möller is lost, a neotype for Saccoblastia sphaerospora Möller is proposed. This Costa Rican collection represents the first record since the discovery of the species in 1891. Hyphoderma argillaceum (Bres.) Donk is reported for the first time from Costa Rica as the fungal host of Occultifur internus.
\end{abstract}

Key words: Basidiomycetes, Atractiellales, Auriculariales, Exidiaceae, Sirobasidaceae, Tremellaceae, Tremella, Exidiopsis, Heterochaete, Helicogloea, Saccoblastia, Sirobasidium, Occultifur, Hyphoderma, mycota, neotropics, Costa Rica.

Esta es la tercera entrega sobre basidiomicetos resupinados costarricenses (Kisimova et al. 1997a,b) y en ella se comunican varias especies raras o nuevas para el país o para la ciencia.

\section{MATERIALES Y MÉTODOS}

Los métodos utilizados en el estudio e identificacion de estos materiales se describen adecuadamente en nuestros dos trabajos anteriores, arriba citados. Los ejemplares identificados se depositan en los Herbarios de la Universidad de Costa Rica (USJ) y de Franz Oberwinkler (FO), en la Universidad de Tubinga, Alemania,

\section{Tremellaceae.}

Tremella coalescens L.S. Olive, Mycologia 43: 678. 1951 (Fig. 1).

Cuerpo fructífero en fresco se parece, externamente, a una especie pequeña de Exidia, de $1.5 \times 0.2-0.3 \mathrm{~cm}$, de consistencia firme gelatinosa, de superficie lisa y bordes diferenciados, de color marrón oscuro o rojizo hasta muy oscura y nigricante. En seco el hongo aparece como una película negra muy adherida al sustrato.

Estructura microscópica: Basidios tremeloideos, 18 - $26 \mu \mathrm{m}$ de largo, subgloboso-globosos. Hifas generativas de $2.5-5 \mu \mathrm{m}$ de ancho, hifidias abundantes de 2 - $3 \mu \mathrm{m}$ de ancho, 
todas con fíbulas muy visibles y frecuentemente arqueadas. Hifas haustoriales muy delgadas ( $1 \mu \mathrm{m}$ de ancho), frecuentes en el himenio y subhimenio. Hifas e hifidias maduras o senescentes con paredes engrosadas y contenido oscuro, negruzco. Los basidios son 2-celulares,

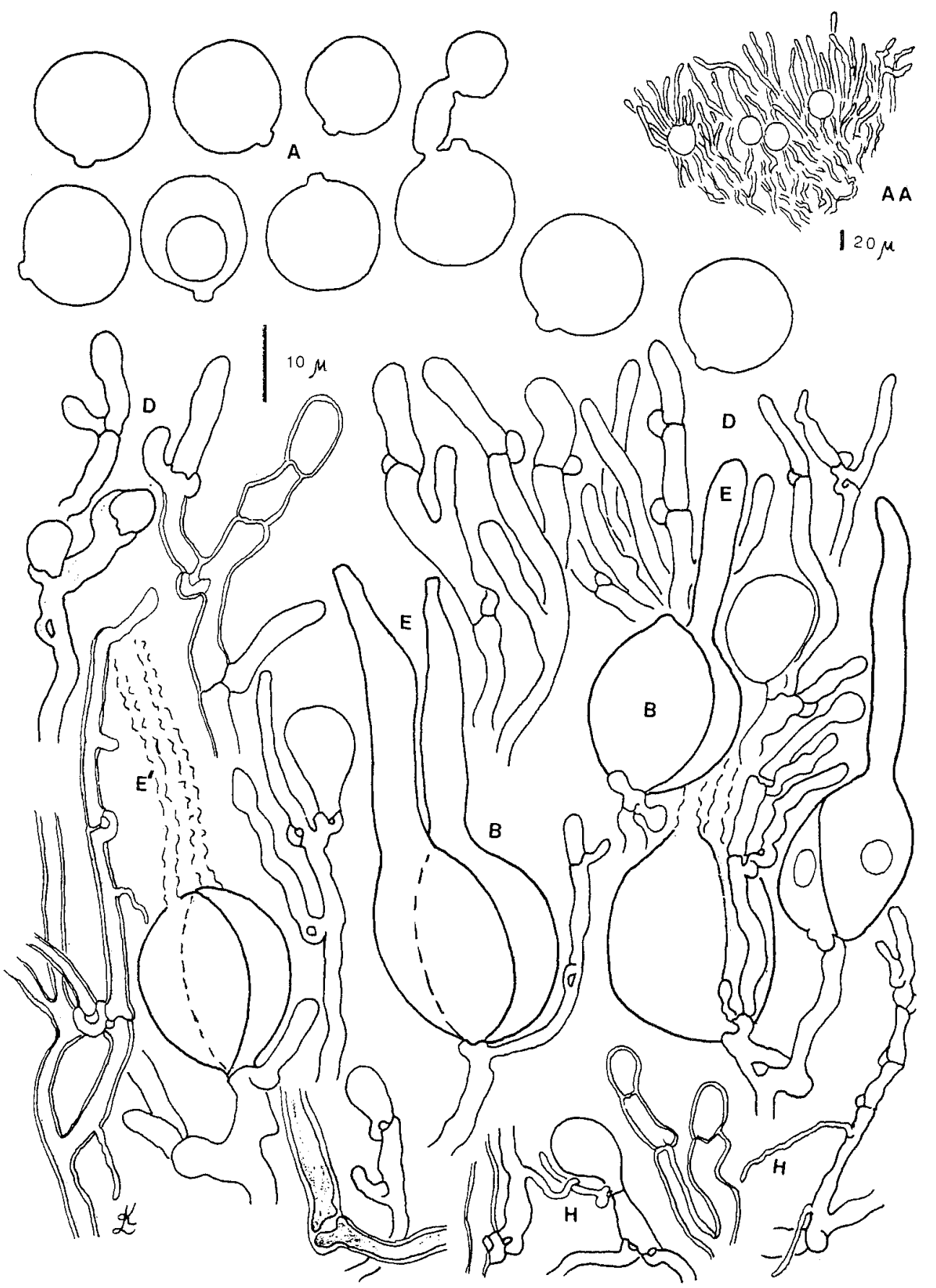

Fig. 1. Tremella coalescens. aa- fructificación en sección transversal. La barra mide $20 \mu \mathrm{m}$. A- basidiosporas, una germina por repetición; B- basidios; D- dendrofisas; E- esterigmas; E'- esterigmas viejos, colapsados; H- haustorios. 
18 - $26 \times 17.5-22 \mu \mathrm{m}$, con esterigmas largos, cilíndricos, $4.5-6 \times 35-40 \mu \mathrm{m}$. Los basidios caducos son de color negro, no se colapsan posteriormente a la esporulación sino que mantienen su forma globosa, solo los esterigmas se colapsan y gelifican. Esporas subgloboso-globosas, de $12-16 \mu \mathrm{m}$, la esporada es blanca.

Material estudiado: Sobre tronco no identificado en suelo, Reserva de Monteverde, Puntarenas, aprox. $1200 \mathrm{~m}, 31 / 12 / 1995$, LKH 275-v (USJ 54525).

Tremella coalescens se parece por el color a $T$. nigrifacta Bandoni \& Carranza, pero difiere de esa especie por su estructura microscópica que revela hifidias abundantes, sus basidios 2-celulares y las esporas de mayor tamaño. Chen (1998), ren su detallada investigación del género Tremella, coloca a $T$. coalescens en el grupo de T. mesenterica, mientras que T. indecorata y T. muriformis, muy semejantes a $T$. nigrifacta, constituyen otro grupo filogenéticamente distinto (y sobre otras especies costarricenses $c f$. Bandoni et al. 1996).

\section{Sirobasidiaceae \\ Sirobasidium minutum Kisim., Oberw. \& L.D.Gómez, sp. nov. (Fig.2).}

Basidioma minuta, pustuloso-gelatinosa, . albo grisacea. Hyphae hyalinae, 3 - 4 um diam., fibulatae, hyphis basalibus ramificatis. Hyphidia nulla. Basidia aovato-elliptica, $10-17 \times 17$ $20(25) \mu \mathrm{m}, 2$-cellulata, concatenata hyphaeque intercalata. Sterigmata fusiformia, 5 - $6 \times 15$ - 20 um. Sporae globosae, 5 - $5.4 \mu \mathrm{m}$, minutissimae apiculatae.

Cuerpo fructífero fresco en forma de pequeñas pústulas gelatinosas, blanco ceniciento, $0.5-1.5 \mu \mathrm{m}$. En seco son casi invisibles, hialinas, reducidas a una película lustrosa. Hifas hialinas o algo translúcidas, de paredes delgadas, 3 - $4 \mu \mathrm{m}$ de diámetro, fibuladas. Hifas basales ramificadas, las generativas no ramifica- das, hifidias ausentes. Basidios ovalado-elipsoidales, $10-17 \times 17-20(25) \mu \mathrm{m}$, con fíbula basal, 2-celulares con un septo oblícuo, separados entre sí por un segmento de hifa generativa de $6-12 \mu \mathrm{m}$ de longitud. Esterigmas (epibasidios, protosterigmas) largamente ovalados, fusiformes, 5 - 6 x 15 - $20 \mu \mathrm{m}$. Esporas globosas, 5 - $5.4 \mu \mathrm{m}$, diminutamente apiculadas.

Material estudiado: Sobre ramilla en suelo, Estación Las Alturas, Coto Brus, Puntarenas, aprox. 1600 m, 29/12/1996, LKH 154-vi (USJ 55485). Holotypus!

Etimología: del latino minutum, pequeño, por el reducido tamaño del basidioma. Nuestra nueva especie difiere de todas las especies conocidas de Sirobasidium Lagerh. \& Pat., por la disposición de los basidios con intercalación de segmentos grandes de la hifa generativa. Una sola especie de Borneo y Java, Sirobasidium magnum Boedijn, según la ilustración original de Boedijn (1934), presenta un arreglo de los basidios semejante a $S$. minutum pero difiere de la nueva especie por ser de mucho mayor tamaño $(4-7 \mathrm{~cm})$ que la asemeja a Tremella foliacea Pers., y es de color marrón hasta marrón rojizo

El género Sirobasidium es de los tremelales el más típicamente tropical. Sus características son los basidios 2-celulares y de septo oblícuo dispuestos secuencialmente, uno tras otro, en una hifa generativa. El basidio apical es el más maduro y seguida la esporulación se colapsa y gelifica. Los esterigmas son firmes, relativamente cortos, fusiformes y se desprenden del basidio generalmente antes de la aparición de esporas globosas y apiculadas más pequeñas que las normales, de ahi que muchos investigadores llamen a esas esporas " basidiosporas sésiles" y a las esporas apiculadas como "esporas secundarias" Aqui utilizamos la terminología de Bandoni (1957) y Lowy $(1956,1977,1980)$ refiriéndonos a esas estructuras como esterigmas y esporas, respectivamente. 


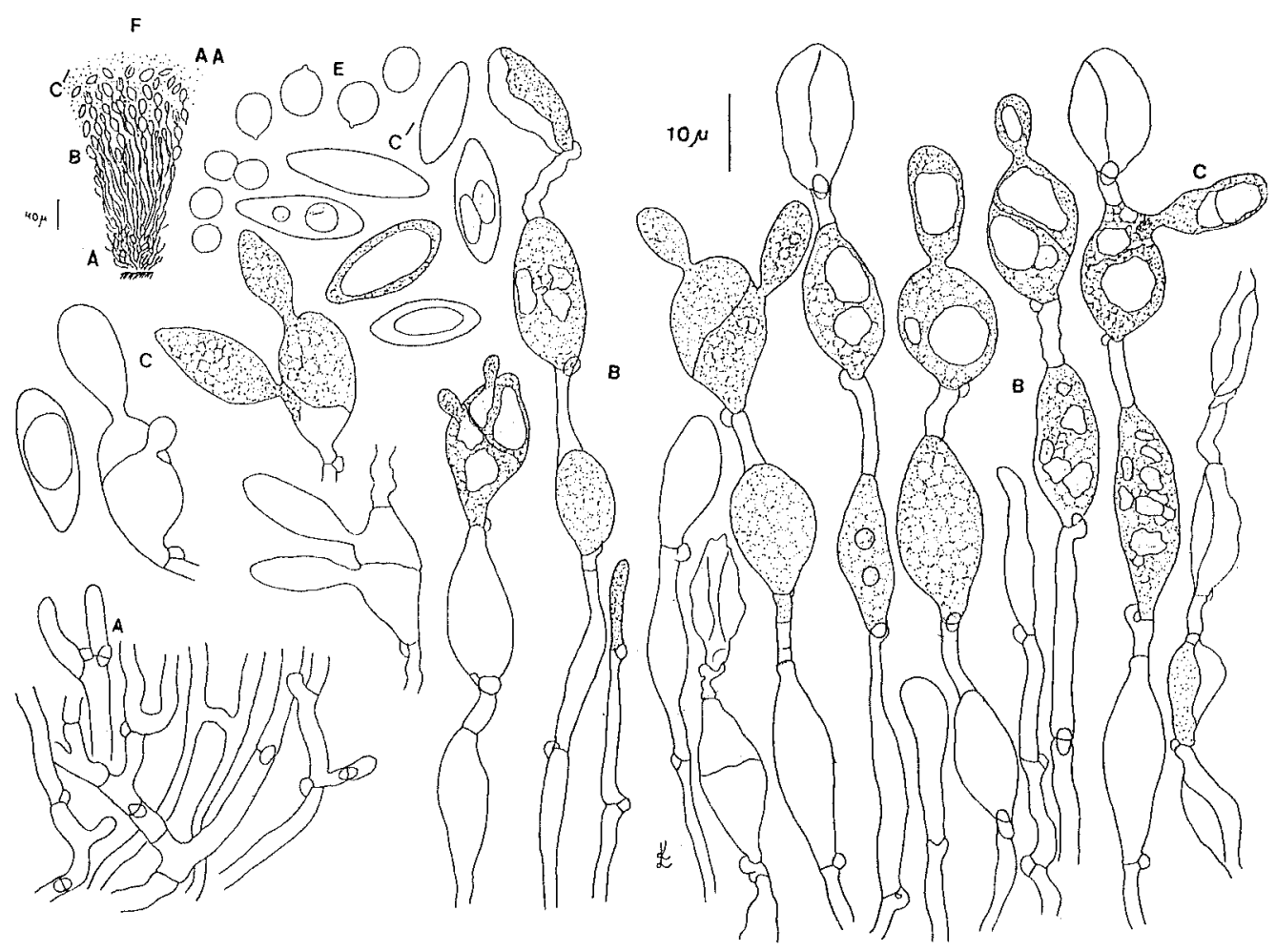

Fig. 2. Sirobasidium minutum. AA- basidioma en sección transversal; A- hifas basales; B- basidios; C- esterigmas; E'- esterigmas separados del basidio; E - esporas apiculadas.

\section{Exidiaceae}

Heterochaete vitrea Kisim., Oberw. \& L.D. Gómez, sp. nov. (Fig. 3).

Fructificatio humida et viva maculosa albo-grisacea, 3 - $10 \mathrm{~mm}$ lata. Clavae cylindrico-fusiformiae, $100-170 \mu \mathrm{m}$, non-septatae, efibulatae, hyalinae, siccu vitreae. Hyphae hyalinae, 2 - 3 um diam., fibulatae, convolutae. Gloeocystidia nulla. Basidia subglobosoglooasa, 13 - $17 \times 14$ - $18 \mu \mathrm{m}$, 4-cellulata. Sterigmata subcylindrica, flexuosa. Sporae aovato-ovatae, pauciter adaxio-lateraliter compressae, 7 - $9 \times 10-12 \mu \mathrm{m}$, apiculatae.

La fructificación fresca es una costra delgada y fina, blanco-grisácea, algo gelatinosa, de 3 - $10 \mathrm{~mm}$ de alto. Sub lente, la superficie parece espinosa por la presencia de abundantes clavijas ("pegs") hialinas y lustrosas. En seco, el hongo es casi invisible al ojo. Clavijas cilín- dricas, ahusadas, $100-170 \mu \mathrm{m}$, de hifas estériles y sin septos, sin fíbulas, hialinas, de 3 - 4 $\mu \mathrm{m}$ de diámetro, con paredes engrosadas también hialinas, en fresco flojamente adherentes entre sí y en seco aglutinadas, siempre muy refractarias y en apariencia vidriosas. En la mitad inferior y parte media de las clavijas se observan delgadas hifidias (dendrofisas) sólo visibles en preparaciones de material fresco o fijado en alcohol acético. Himenio muy delgado, $30-40 \mu \mathrm{m}$, monomítico, con hifas hialinas y retorcidas, de 2 - $3 \mu \mathrm{m}$ de diámetro. Fíbulas opacas. Gloeocistidios ausentes. Basidios subglobosos o globosos, 13 - 17 x 14 - $18 \mu \mathrm{m}$, 4celulares, los involucros de los basidios caducos son muy visibles. Esterigmas subcilíndricos, flexuosos, 3 - 4 x 13 - $15 \mu \mathrm{m}$. Esporas ampliamente ovalado-aovadas, algo comprimidas adaxiolateralmente, 7 - 9 x $10-12 \mu \mathrm{m}$, con un apículo conspicuo. 

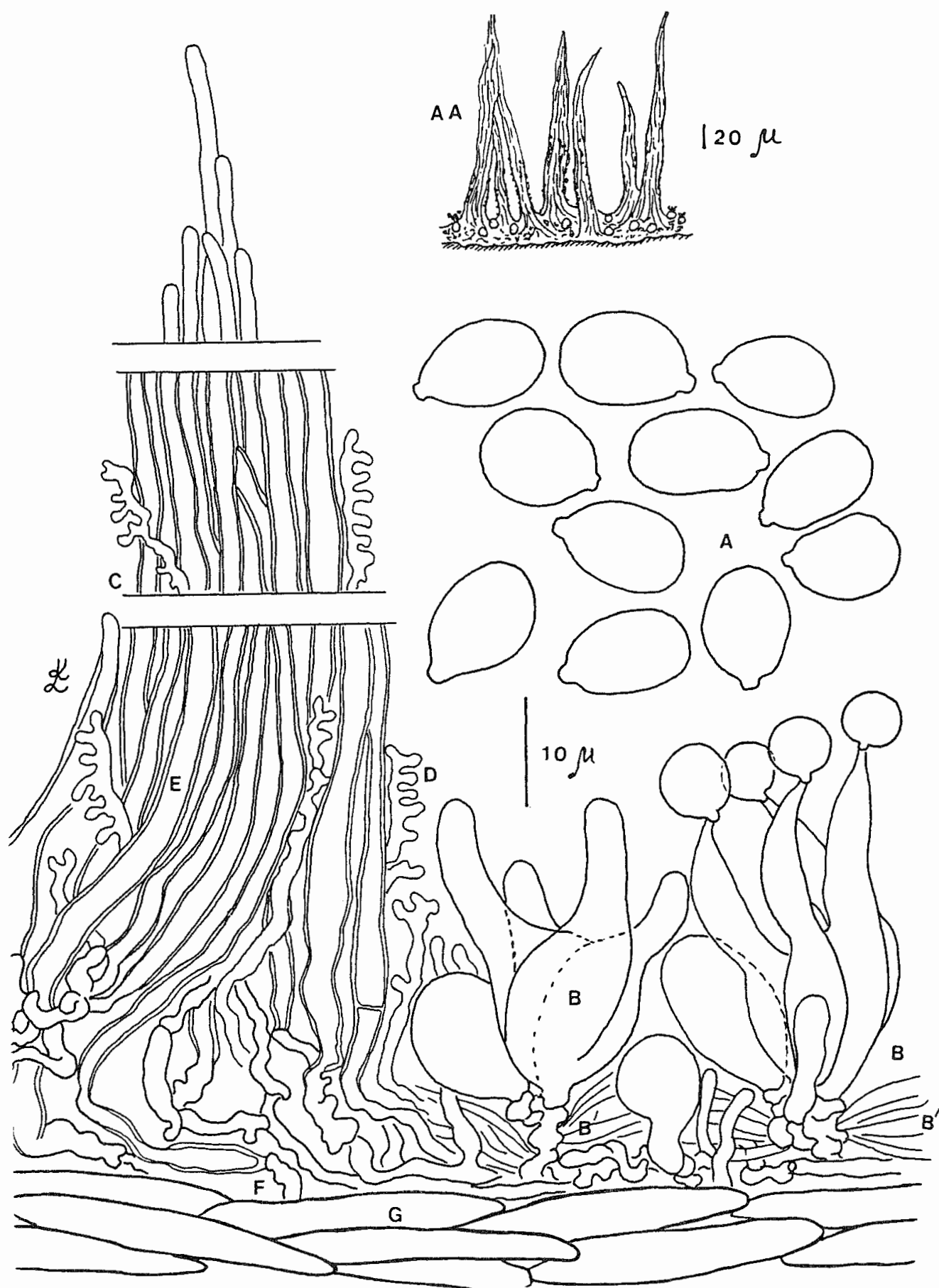

Fig. 3. Heterochaete vitrea. AA- fructificación en sección transversal; A- esporas; B - basidios; C - clavijas (pegs); D - dendrofisas; E - hifas de la clavija; F - hifas basales; G - sustrato. 
Material estudiado: Sobre ráquides herbáceos muertos, de helecho. Reserva Biológica de San Ramón, Alajuela, 1100 m, 27/12/1996, LKH 305-vi (USJ 55487), Holotypus!

Etimología: Del latino vitreum, vidrio, vidrioso, por el brillo y refracción de las clavijas.

Heterochaete vitrea difiere de todas las especies descritas por el sustrato herbáceo y no leñoso, por sus clavijas muy delgadas, hialino-vidriosas y por la relación de largo : ancho de sus esporas: 1.3 - 1.4. Además esta nueva especie, como indicamos en la descripción, es monomítica. De otras especies con un solo tipo de hifas difiere, en la relación largo:ancho de las esporas (L:A) de la forma siguiente: $H$. roseola Pat., descrita de Hanoi, Vietnam, por las clavijas marrón, obtusas, basidios ovalados y esporas más alargadas (L:A $>2$ ). De $H$. minuta Pat. del Ecuador y de $H$. minuta $f$. congolensis Bresadola, de CongoKinshasa, por las clavijas mucho más largas, hasta $450 \mathrm{~mm}$, y las esporas reniformes (L:A $>2$ ). De las especies dimíticas, además de la particular estructuración del basidioma: $H$. hirneoloides $\mathrm{K}$. Wells (sin. H. tremellispora (Möll.) Bodman), tiene el cuerpo fructífero y las esporas más grandes. De $H$. mussoriensis Bodman, descrita del Punjab, India, y de $H$. lividofusca Pat., de Ecuador, porque tienen mayores dimensiones en todas sus estructuras y basidiomas y L:A $>2$.

Las especies costarricenses de Heterochaete Pat. que suman con esta cuatro, han sido revisadas en nuestra publicación anterior (Kisimova et al. 1997b y cf. Bodman 1952).

Exidiopsis mucedinea (Pat.) K.Wells, Lloydia 20: 46. 1957. (Fig. 4)

(Sebacina mucedinea Pat., Herb. Boiss. 3: 60. 1895; Exidiopsis manihoticola Viegas, Bragantia 3: 23. 1943; Thelephora mucedinea (Pat.) Sacc. Syll. Fung. 14: 214. 1899).
Cuerpo fructífero de poco espesor, en columnas, ceroso-gelatinoso, de color gris a marón pálido, el margen indiferenciado, muy delgado y fino, con superficie pruinosa, visto con lente la superficie presenta numerosas papilas blancas. Las papilas, de $40-70 \mu \mathrm{m}$ de largo, están formadas por una masa amorfocristalina blanca y de esporas. La fructificación en seco es una pelicula más pálida que en fresco y con las papilas muy blancas. Hifas hialinas, delgadas, de $2-2.5 \mu \mathrm{m}$ de diámetro, con fíbulas pequeñas, a veces arqueadas. Hifidias (paráfisas) son numerosas, más delgadas que las hifas, ramificadas $y$, a veces, se desintegran y dan a la superficie una apariencia finamente granulosa, amarillenta. Probasidios ovoides o piriformes, con fíbulas basales a menudo arqueadas. Basidios 4-celulares, ovoideo-globosos, 10.5 - 14.5 x 8 - 12(16) $\mu \mathrm{m}$. Esterigmas largos, flexuosos. Esporas cilíndricocurvas o elípticamente comprimidas, 8 - $10 \mathrm{x}$ $3.5-5 \mu \mathrm{m}$.

Material estudiado: Reserva Biológica de San Ramón, Alajuela: 2/12/1993, LKH 5-iv (USJ 55348, FO 44 674); 28/12/1996, LKH 315-vi (USJ 55488).

Exidiopsis mucedinea difiere de otras especies tropicales que presentan aglomeraciones de cristales en el himenio, v.g. Exidia tucumanensis Lowy, porque esta última especie presenta una fructificación que seca de color negro y esporas cilíndricas (12 - 15 x $5.5-6$ $\mu \mathrm{m})$ y de Exidiopsis macroacantha K. Wells porque esta presenta los cristales sobre gloeocistidios y sus esporas son elípticas o subglobosas $(7-8.5 \times 5-7 \mu \mathrm{m})$.

En el material costarricense no observamos gloeocistidios como los que describe Olive (1951), pero si parafisoides. El tipo de Exidiopsis mucedinea fue descrito de Ecuador y la especie se conoce de Panamá ( Martin 1944), El Salvador (Wells 1961), Brasil (Viégas 1943) y Tahiti (Olive 1958). 


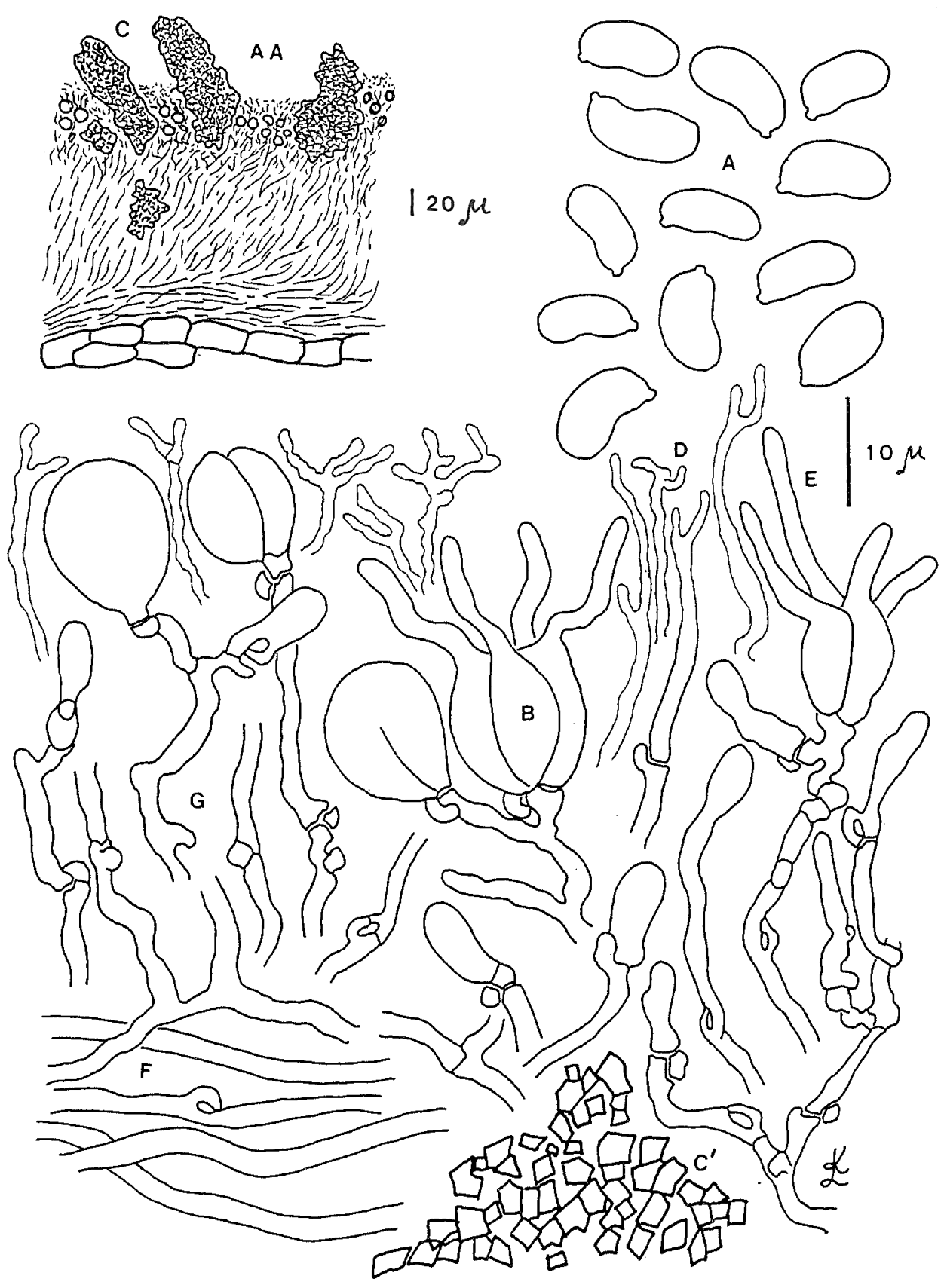

Fig. 4. Exidiopsis mucedinea. AA - fructificación en sección transversal con C- papilas cristalinas sobre el himenio; A - esporas; B - basidios; C'- cristales en papilas; D - dendrofisas; E - esterigmas; F - hifas basales; G - hifas del himenio. 


\section{Atractiellales (Auriculariales s.l.).}

Las siguientes tres especies, de los géneros Helicogloea Pat. Saccoblastia Möller y Occultifur Oberw,. tienen basidios cilíndricos, transversalmente septados y pertenecen, según Jülich (1981) al orden Auriculariales. Sin embargo, se distinguen significativamente del tipo Auricularia por los poros septales simples y otras características ultraestructurales así como por razones moleculares que hacen difícil la sistemática del grupo (Oberwinkler \& Bauer 1989), y que aquí asignamos al orden Atractiellales.

\section{Helicogloea Pat., Bull. Soc. Mycol. Fr. 8:} 121. 1892.

Fructificaciones en fresco gelatinosas, en seco ceroso-córneas. Hifas relativamente delgadas, 2.5 - $4 \mu \mathrm{m}$, sin fíbulas. Probasidios con sacos péndulos ( ámpulas, ampollas) y doblados hacia el sustrato, intercalados en las células basales. Basidios largamente cilíndricos, curvos en algún grado, con esterigmas relativamente anchos y apicalmente aguzados. Esporas ovoides que germinan con hifas o por repetición. El tipo del género es Helicogloea lagerheimii Pat.

Helicogloea aurea Baker, Mycologia 38: 635. 1946. (Fig. 5).

Esta especie se distingue de las otras del género por el color anaranjado-amarillento mate de la fructificación seca, que en fresco es de color grisáceo blanquecina. Los sacos probasidiales son muy largos, $30-50 \mu \mathrm{m}$, y las muy largas células basales del basidio que miden $100-200 \mu \mathrm{m}$. La parte fértil del basidio, de $40-60 \times 8-12 \mu \mathrm{m}$, es 4-celular, con esterigmas subagudos, 8 - $10 \times 2.5-3 \mu \mathrm{m}$ esporas ovoides, $15-20 \times 6.5-10 \mu \mathrm{m}$.

Material estudiado: Estación Biológica La Selva, Puerto Viejo, Sarapiquí, Heredia, aprox. 30 m, 25/12/1995, LKH 226-v (USJ 54483).

Esta especie era conocida solo del ejemplar tipo procedente de Panamá, recogido por Martin en 1935 (Baker 1946).
Saccoblastia Möller, Protobasidiomyceten, $162,1895$.

Es un género muy discutido cuyas características principales, según la descripción original, son el reducido tamaño de las fructificaciones, de hasta $1 \mathrm{~mm}$ y que son enrejados de hifas. Los basidios solitarios y la presencia del saco o ámpula en la célula que subtiende al probasidio cuyo contenido celular contribuye al desarrollo del basidio.

El género fue descrito con dos especies muy distintas entre sí, recolectadas en la Estación Blumenau, Brasil: Saccoblastia ovispora Möller recogida en 1892 y S. sphaerospora Möller recogida en 1891. Sin embargo, en su publicación Möller (1895) no indicó cuál era la especie típica. A continuación una descripción sucinta de cada especie según el texto de Möller:

Saccoblastia ovispora Möller, Protobasidiomyceten 162. 1895.

Fructificaciones gelatinosas. Hifas sin fíbulas. Sacos probasidiales piriformes, $30 \times 8$ $\mu \mathrm{m}$. Basidios largos, de hasta $100 \mu \mathrm{m}$, con esterigmas de forma de punzón y que salen de la parte media de cada célula basidial. Esporas como en Exidia Fr., 13 x $7-8 \mu \mathrm{m}$, germinan por repetición o con hifas y que se dividen transversalemnte en 2 - 3 células. Las esporas, así como los ápices de algunas hifas, forman pequeños conidios redondos.

\section{Saccoblastia sphaerospora Möller, Protobasi-} diomyceten 162. 1895.

Fructificación flocoso-hipocnoidea, con hifas de mayor diámetro que las de $S$. ovispo$r a$, con grandes fíbulas muy conspicuas y numerosas. Sacos probasidiales globosos, de $11 \mu \mathrm{m}$. Basidios más cortos, $45-60 \mu \mathrm{m}$, con esterigmas muy delgados, filiformes, que salen de cada célula basidial en su parte más extrema apical. Esporas globosas, de 6 - $8 \mu \mathrm{m}$, que germinan con hifas, no se dividen o forman conidios. 


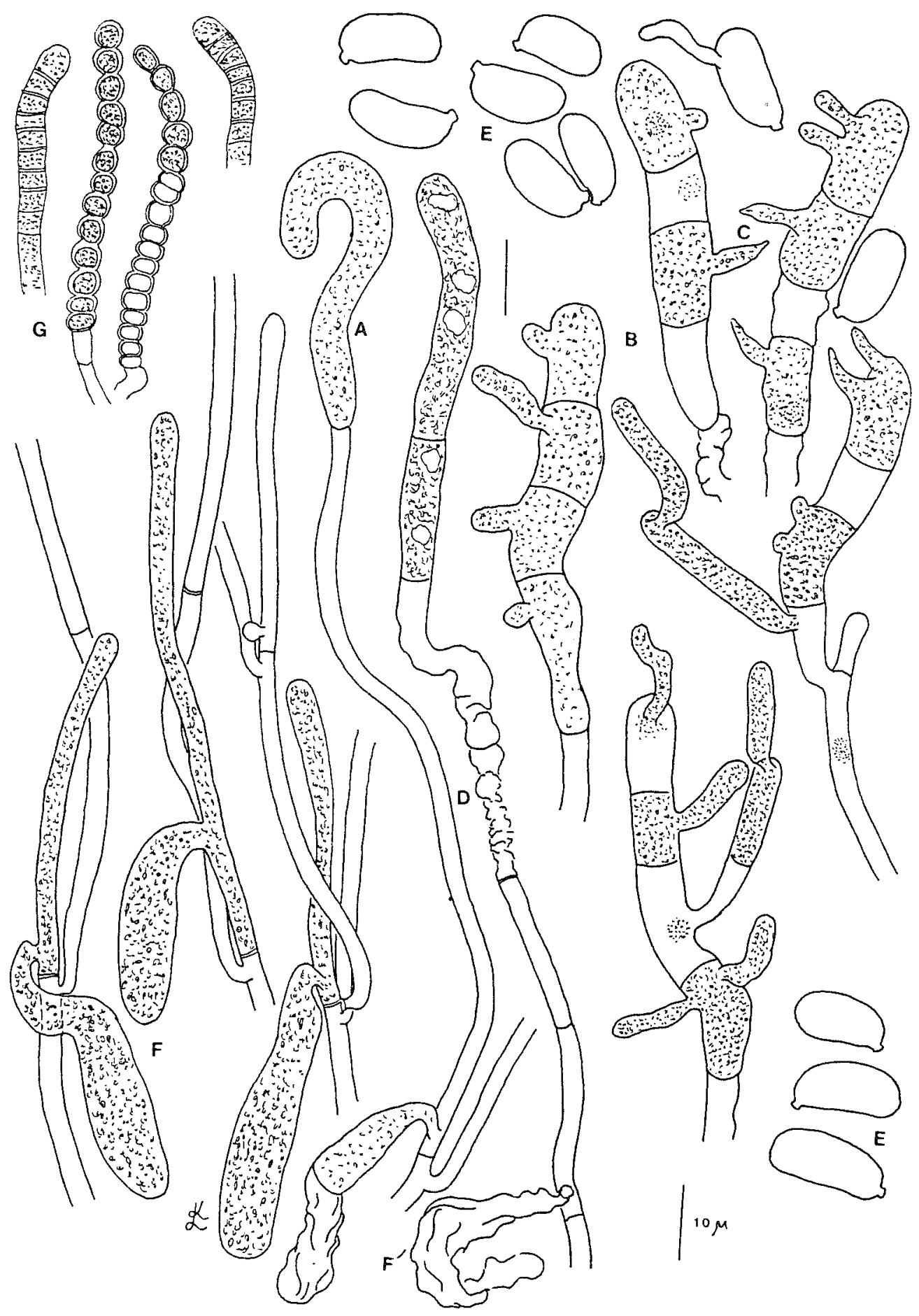

Fig. 5. Helicogloea aurea. A - probasidio; B - basidio; C - esterigmas; D - célula basal del probasidio; E - esporas; F - sacos o ámpulas basidiales; $\mathrm{F}^{\prime}$ - saco basidial vacio; $\mathrm{G}$ - conidios en extremos de hifas vegetativas. 
Möller admite, en la publicación original, que $S$. ovispora es semejante a Helicogloea lagerheimii Patouillard, descrita de material ecuatoriano tres años antes de su hallazgo en Brasil, pero cuya descripción consideró insuficiente para asignarla a la especie de Patouillard.

Es necesaria alguna discusión sobre este interesante género. Bourdot \& Galzin (1927) admitieron el género Saccoblastia y distinguieron dos subgéneros: 1- Saccoblastia con fructificaciones membranáceas y floculosas y presencia de fíbulas y, 2- Saccogloea con fructificaciones mucoso-gelatinosas y ausencia de fíbulas.

En los años siguientes aparecieron descritas varias especies de ambos tipos pero $S$. ovispora con frecuencia fue mantenida como el tipo de género, a pesar de que Möller no lo indicara así. Baker $(1936,1946)$ sinonimizó Saccoblastia con Platygloea Pat. , nombre que había sido reemplazado por el de Helicogloea, e hizo nuevas combinaciones de todas las especies descritas de Saccoblastia como representatentes de Helicogloea además de errar al indicar que $S$. sphaerospora no presentaba fíbulas por no haber visto el material tipo ni existir nuevas colecciones.

Donk (1966) opinó que Helicogloea y Saccoblastia eran dos géneros discretos y diferentes y sugirió la necesidad de nuevas colecciones tropicales para esclarecer el problema. Lowy (1971) reconoció Saccoblastia como un subgénero, pero también ex lit. y sin tener a la vista el material de Möller o nuevas colecciones, recombinó S. sphaerospora como Helicogloea sphaerospora (Möller) Lowy.

Más tarde, reapareció Saccoblastia en la literatura (Wojewoda 1977, Jülich 1981, Oberwinkler \& Bauer 1989, Bauer \& Oberwinkler 1997) sin embargo no habían a la mano nuevas colecciones ni el material original de Möller.

Dos colecciones costarricenses corresponden a la descripción e ilustración de Möller y por esa razón las asignamos a Saccoblastia sphaerospora Möller, y constituyen los primeros registros de Saccoblastia desde su hallazgo por Möller.
Saccoblastia sphaerospora Möller, Protobasidiomyceten 162, 1895. (Figs. 6, 7, 8).

Fructificación inicialmente de pequeñas manchas costrosas, $0.5-1 \mathrm{~mm}$, que posteriormente se expanden y confluyen hasta cubrir unos centímetros cuadrados de superficie, en fresco blanco grisácea, hidnoide-reticulada. En seco es flocoso-poroide o hipocnoide, blanco amarillenta. Hifas del subículo con paredes engrosadas, 4.5 - $7 \mu \mathrm{m}$ de diámetro; en el subhimenio 3 - $5 \mu \mathrm{m}$ diám., con paredes delgadas. Fíbulas grandes y conspicuas. Sacos probasidiales ovalados, 12 - $15 \times 8-10 \mu \mathrm{m}$, basales en las células primordiales, cercanos a la fíbula. Basidios 50 - $80 \times 5-6.5 \mu \mathrm{m}$, con esterigmas muy delgados, filiformes, 4 - $5 \mu \mathrm{m}$ de largo, en cada célula basidial cerca del septo con la célula siguiente, excepto en la última célula (apical) en cuyo caso los esterigmas son apicales. Esporas globosas, $8-10 \mu \mathrm{m}$, con apículo prominente, germinan con hifas o por repetición. No se observaron conidios en el material costarricense.

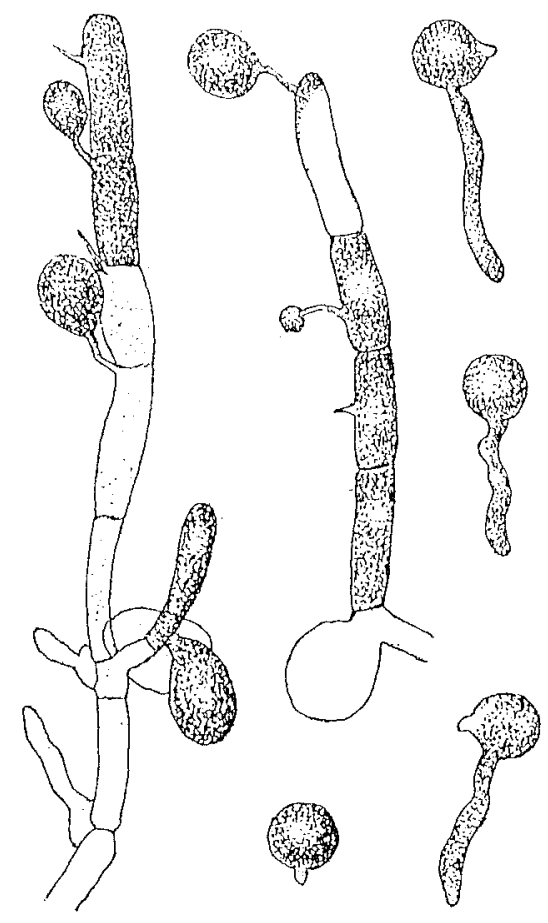

Fig. 6. Saccoblastia sphaerospora Möller. Reproducido del original (Möller 1895) 
Material estudiado: Estación Biológica La Selva, Sarapiquí, Heredia, en bosque lluvioso primario. 14/12/1993, LKH 115-iv (USJ 54699; FO 44780). Estación Biológica Las Al- turas, Coto Brus, Puntarenas, $1500 \mathrm{~m}$, 2/12/1996, LKH 222-vi (USJ 55489). El hábitat de La Selva es similar al de la localidad típica: Blumenau, Brasil.

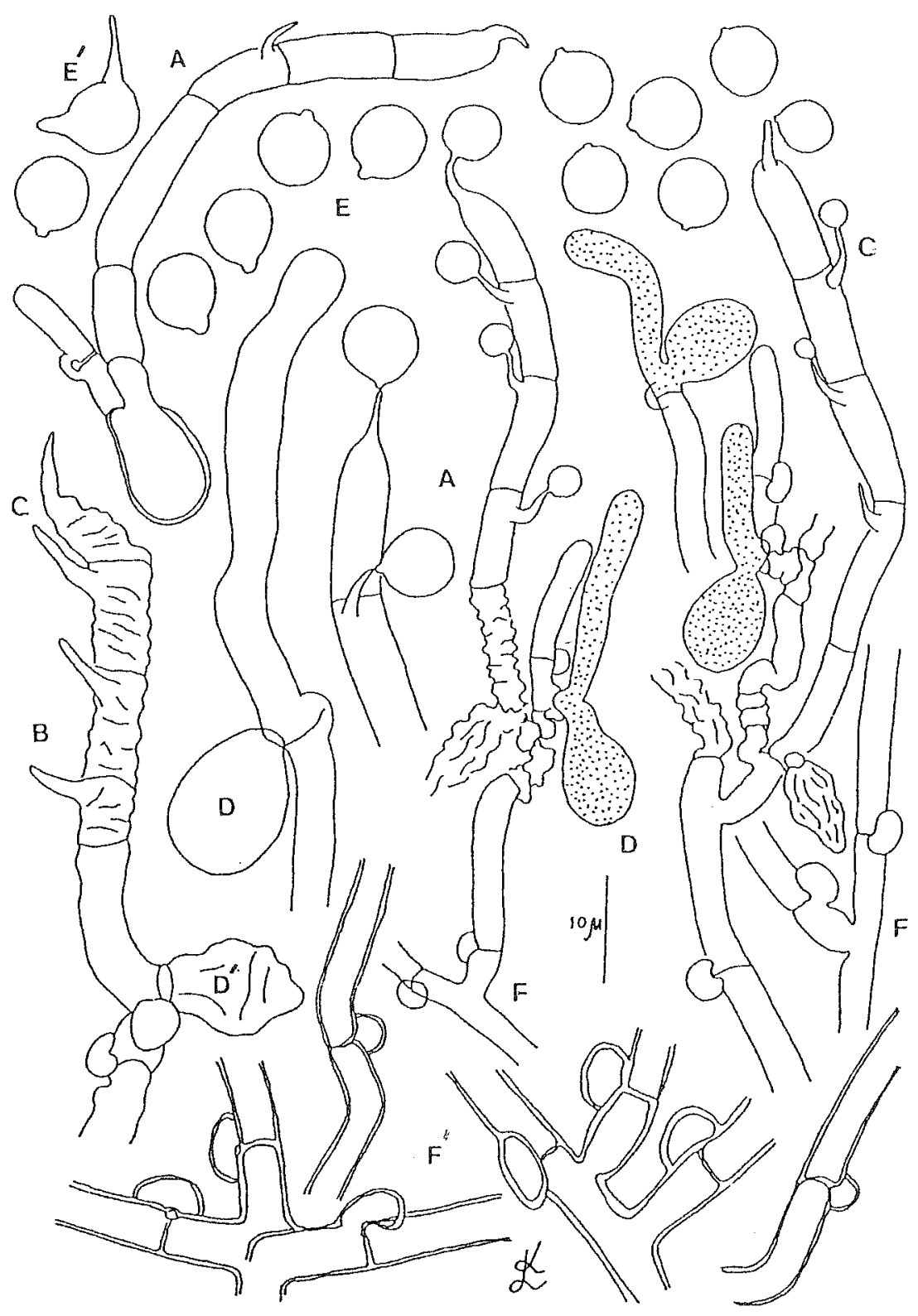

Fig. 7. Saccoblastia sphaerospora. A - basidio; B - basidio colapsado; C - esterigma; D - saco probasidial; D'- saco vacio; E - esporas; $E^{\prime}$ - espora que germina por repetición; F - hifas del subhimenio; $F^{\prime}$ - hifas del subículo. El ejemplar ilustrado es LKH 115 -iv (USJ 54699), procedente de Estación La Selva, Heredia. 


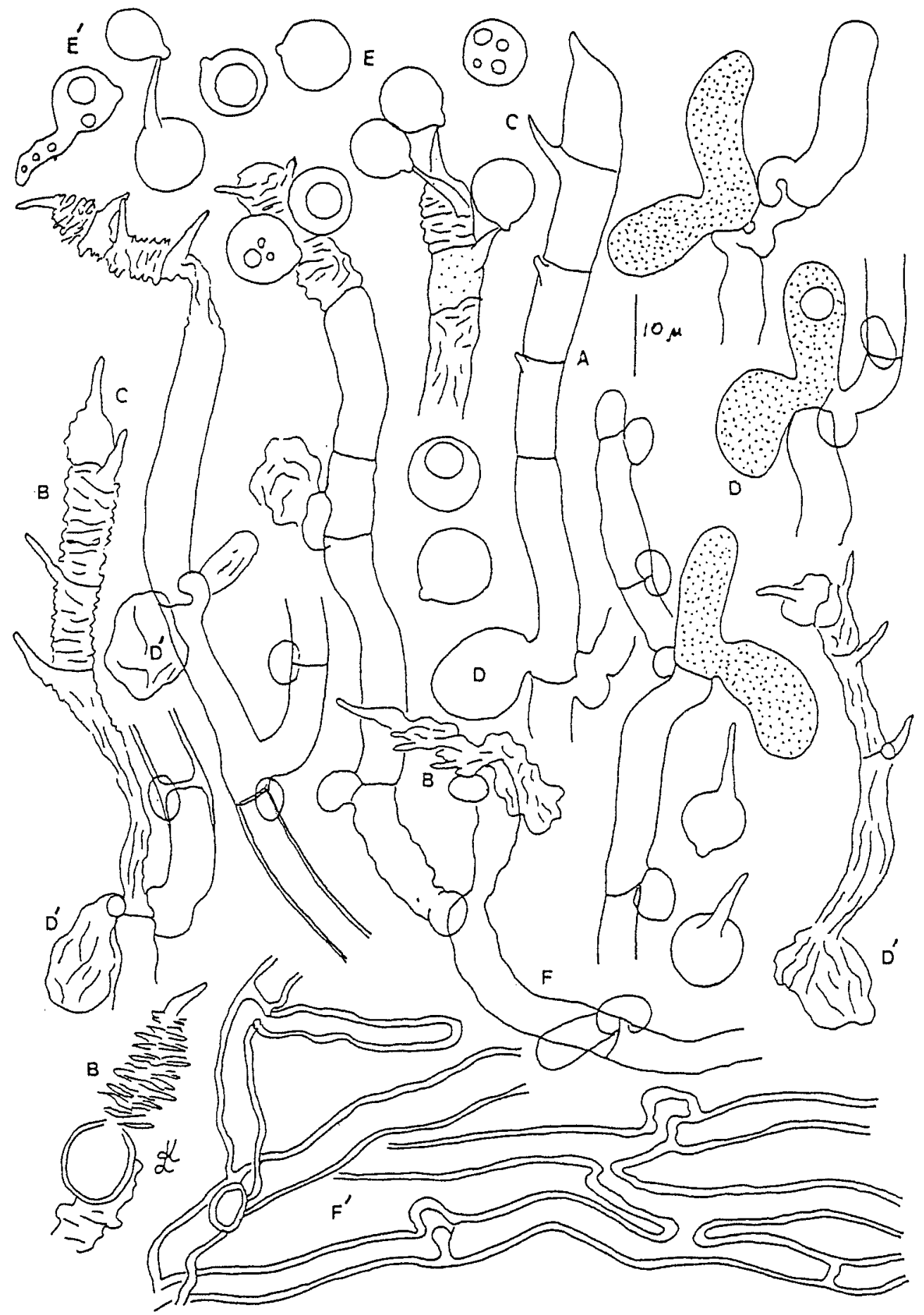

Fig. 8. Saccoblastia sphaerospora. A - basidio; B - basidio colapsado; C - esterigma; D - saco probasidial; D'- saco probasidial vacio; E - esporas; E'- esporas que germinan por repetición y por hifas; $F$ - hifas del subhimenio; $F^{\prime}$ - hifas del subículo. El ejemplar ilustrado es LKH 222-vi, de Las Alturas, Coto Brus, Puntarenas que se propone como neotipo del género y de la especie de Möller. 
Las colecciones originales de Möller o sus dibujos originales ya no existen (Friedrichsen, 1977). El Código Internacional de Nomenclatura Botánica en su Artículo 9.3 estipula que el holotipo puede ser una ilustración. En ausencia de ilustraciones originales de Möller el único material gráfico con la sanción del autor es la ilustración que aquí se reproduce (Fig. 6) que, sin embargo, no es única. Por ese motivo, proponemos aquí la erección de un neotipo en conformidad con el Artículo 7.8 del mencionado Código y, para ese efecto, señalamos nuestra colección procedente de Estación Biológica Las Alturas, Coto Brus, Puntarenas LKH 222-vi (USJ 55489) como neotipo de Saccoblastia sphaerospora Möller, como neotipo del género Saccoblastia
Möller, y a la especie $S$. sphaerospora Möller como especie tipo de este curioso y extremadamente raro género.

Otro representante de este grupo de hongos auricularioides de septos con poros simples es el género monotípico Occultifur Oberwinkler, también representado en nuestro material costarricense:

Occultifur internus (L.S. Olive) Oberw., Rep. Tottori Myc. Inst. 28:121. 1954. (Fig. 9).

(Platygloea interna L.S. Olive, Bull. Torrey Bot. Club 85: 5-27. 1958).

Género y especie parásitos en el himenio de otros basidiomicetes resupinados y en hongos del grupo de los Dacrymycetales.

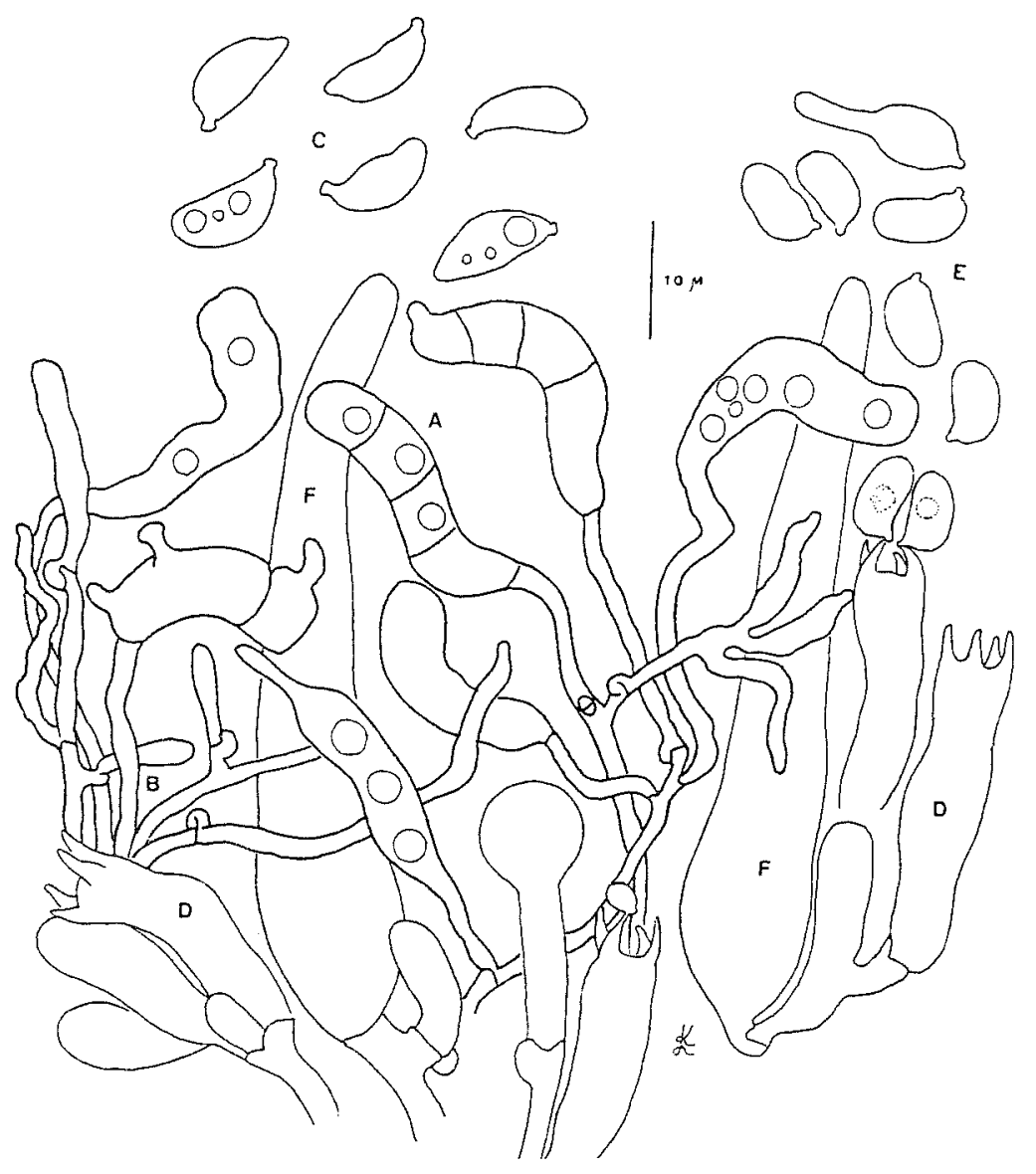

Fig. 9. Occultifur internus en tejidos de Hyphoderma argillaceum. A - basidio; B - hifas; C - esporas. D - basidios de H. argillaceum; E - esporas de H. argyllaceum; F - cistidios de H. argillaceum. 
Hifas delgadas, 1 - $2 \mu \mathrm{m}$, con fíbulas. Basidios cilíndricos, en racimos o fascículos, con largas células basales. Parte fértil del basidio 25 - 30 x 5 - $6 \mu \mathrm{m}$. Esporas almendradas, 10 $11 \times 3.5-5 \mu \mathrm{m}$, con un apículo largo.

Material estudiado: Parásito en Hyphoderma argillaceum (Bres.) Donk, Estación Biológica de San Ramón, Alajuela, aprox. 1500m, 04/03/1992, LKH 59-iii (FO 43957).

El material costarricense difiere de la especie tipo: por el hongo hospedero, basidios más cortos y esporas más largas que, en la especie tipo miden, respectivamente, $45-60$ x 5 - $6 \mu \mathrm{m}$ y $7-8 \mathrm{x}$ $3.5-4 \mu \mathrm{m}$. El hongo hospedero, originalmente descrito como Peniophora argillacea se conocía hasta ahora de Jamaica, Santa Lucía, Dominica, Europa (Punugu et al. 1980), Irán (Hallenberg 1981), Norteamérica (Martin \& Gilbertson 1977), Argentina (Hjortstam \& Ryvarden 1986).

\section{AGRADECIMIENTO}

A las autoridades de la O.T.S. por permitirnos el uso de las estaciones de La Selva y Las Cruces. A las autoridades de la Reserva de San Ramón, Campus de Occidente, por el uso de sus instalaciones. A la Estación Las Alturas y a la Reserva de Monteverde por permitirnos investigar en ellas. A tres revisores anónimos por valiosas sugerencias al manuscrito.

\section{RESUMEN}

Se comunica la presencia en Costa Rica de los siguientes hongos basidiomicetos resupinados: Exidiopsis mucedinea, Helicogloea aurea Baker que hasta ahora solo se conocía del holotipo panameño, Heterochaete vitrea especie nueva, Occultifur internus, originalmente descrita de Tahiti, Saccoblastia sphaerospora Möller, descrita en 1895 de Brasil y desde entonces no recolectada. Se propone esa especie como especie típica del género Saccoblastia y se propone la colección costarricense como neotipo, Sirobasidium minutum, especie nueva, y Tremella coalescen antes conocida solo de E.E.U.U.. Todas las especies se ilustran y pertenecen a varios grupos de Auriculariales s.l.. El penioforoide Hyphoderma argillaceum, hospedero de Occultifur internus, no se conocía hasta ahora de Costa Rica.

\section{REFERENCIAS}

Baker, G. F. 1936. Study of the genus Helicogloea. Annals Missouri Bot. Garden. 23: 69 - 128

Baker, G. F. 1946. Addenda to the genera Helicogloea and Physalacria. Mycologia 38: 630 - 638.

Bandoni, R. J. 1957. The spores and basidia of Sirobasidium. Mycologia 49: 250 - 255.

Bandoni, R.J., Carranza, J., \& A. A. Bandoni. 1996. Four new species of Tremella (Tremellales, Basidiomycotina) from Costa Rica. Rev. Biol. Trop. 44 (Supl. 4): $15-24$.

Bauer, R. \& F. Oberwinkler, 1997. The Ustomycota: an inventory. Mycotaxon 64: 303 - 319.

Bodman, M.C. 1952. A taxonomic study of the genus $\mathrm{He}$ terochaete. Lloydia 15: 193 - 233..

Boedijn, K.B., 1934. The genus Sirobasidium in the Netherlands Indies. Bull. J. Bot. Buitenzorg III, 13: $266-268$.

Bourdot, H. \& A. Galzin. 1927(1928). Hyménomycetes de France, 4 - 5. Bibliotheca Mycologica 13 [Reimpresión 1969].

Chen, Ch.-J. 1998. Morphological and molecular studies in the genus Tremella. Bibliotheca Mycologica 174.

Donk, M.A. 1966. Checklist of European Hymenomycetous heterobasidiae. Persoonia 4: 5 - 335 .

Friedrichsen,I. 1977. The fate of the fungi collected by A. Möller in Brazil (1890 - 1895) And a list of specimens still existing. Mitt. Inst. Alg. Bot. Hamburg 15: 99-104.

Hallenberg, N. 1981. Synopsis of wood-inhabiting Aphyllophorales and Heterobasidiomycetes from N. Iran. Mycotaxon 12: 473 - 502.

Hjortstam, K. \& L. Ryvarden. 1986. Some new and noteworth fungi from Iguazu, Argentina. Mycotaxon 25: 539 - 567.

Jülich, W. 1981. Higher taxa of Basidiomycetes. Bibl. Mycol. 85.

Kisimova-Horovitz,L., Oberwinkler, F., \& L. D. Gómez. 1997 a. Basidiomicetos Resupinados de Costa Rica. Litschauerella, Subulicystidium y Tubulicium. Rev. Biol. Trop. 45: 1311 - 1324. 
Kisimova-H-,L., Oberwinkler, F. \& L.D.Gómez. 1997 b. Basidiomicetos resupinados de Costa Rica. Exidiaceae (Tremellales). Rev. Biol. Trop. 45 (Supl. 4): $1325-1347$.

Lowy, B. 1956. A note on Sirobasidium. Mycologia 48: $324-327$.

Lowy, B. 1971. Tremellales. Flora Neotropica 6. 153 p.

Lowy, B. 1977. A new Tremella with deciduous sterigmata. Mycotaxon 6: 371 - 374.

Lowy, B. 1980. Tremellales Supplement. Flora Neotropica 6 (1980). 18 p.

Martin, G. W. 1944. New or noteworth tropical fungi.III. Lloydia 7: 67-80.

Martin K.J. \& R.L.Gilbertson 1977. Synopsis of woodrotting fungi on spruce in North America. Mycotaxon 6: 43-77.
Möller, A. 1895. Protobasidiomyceten. Bot. Mitt. aus den Tropen 8. Oberwinkler,F. \& R. Bauer. 1989. The systematics of gastroid auricularioid Hetero-basidiomycetes. Sydowia 41: 224 - 256.

Olive, L.S. 1951. Taxonomic notes on Louisiana fungi.III. Additions to the Tremellales. Mycologia 43: 677 - 690 .

Olive, L.S. 1958. The lower Basidiomycetes of Tahiti.I. Bull. Torrey Bot. Club 85: 5-27, 89-110.

Punugu, A., Dunn, M., A. L. Welden. 1980. The Peniophoroid fungi of the West Indies. Mycotaxon 10: $428-454$.

Viégas, A.P. 1943. Alguns fungos da Mandioca. Bragantia 3(2): 21 - 29.

Wells, K. 1961. Studies of some Tremellaceae.IV. Exidiopsis. Mycologia 53: $317-370$.

Wojewoda,W. 1977. Mala Flora Grzybow. II: 214 - 215. 